\title{
MULTIPLIERS IN $L^{p}$ AND INTERPOLATION
}

\author{
BY WALTER LITTMAN
}

Communicated by W. Rudin, April 12, 1965

For functions $u=u(\xi)$ in $\mathscr{D}(R)^{n}$ ( $C^{\infty}$ with compact support) we consider the mapping $K: u \rightarrow v$ defined by $\hat{\theta}(x)=k(x) \mathfrak{u}(x)$. Here $k$ is a measurable function and " $\leadsto$ " denotes the Fourier transform. If $K$ can be extended to a bounded map from $L^{p}$ to $L^{*}$ we say $k \in M_{p}^{*}$. $\left(M_{p}^{p} \equiv M_{p}\right.$.) Elements in $M_{p}$ are called multipliers in $L^{p}$.

Hörmander [3] extended the result of Mikhlin [4] as follows:

TheOREM (HöRMANDER). A sufficient condition that $k(x)$ be a multiplier in $L^{p}$ for all $1<p<\infty$ is that

$$
\operatorname{Sup}_{R>0} R^{-n} \int_{R<|x|<2 R}\left|R^{\alpha} D^{\alpha} k\right|^{2} d x<\infty
$$

for all $0 \leqq \alpha \leqq \nu$ and some $\nu>n / 2$. (Derivatives in (1) are generalized derivatives.)

On the other hand, it is well known that bounded measurable functions are multipliers in $L^{2}$. Hence it seems reasonable to interpolate between these two results to obtain a criterion for $k \in M_{p}$ for values of $1 / p$ in a given range (which must be a symmetric interval about $1 / 2$, see [3].) That is the point of Theorem A.

THEOREM A. If for some $q \geqq 2$

$$
\operatorname{Sup}_{R>0} R^{-n} \int_{R<|x|<2 R}\left|R^{\alpha} D^{\alpha} k(x)\right|^{q} d x<\infty
$$

for all $0 \leqq \alpha \leqq \nu$ and some $\nu>n / q$, then $k \in M_{p}$ for all $p$ satisfying $|1 / 2-1 / p| \leqq 1 / q$. (If suitably interpreted $\alpha$ may be nonintegral; see below.)

A slight improvement is

Theorem B. If for some $q \geqq 2, \beta>0$

$$
\operatorname{Sup}_{R>0} R^{-n} \int_{R<|x|<2 R}\left|R^{\alpha+\beta} D^{\alpha} k\right|^{a} d x<\infty
$$

for all $0 \leqq \alpha \leqq \nu$, some $\nu>n / q$, then

$$
k \in M_{p}^{*} \quad \text { if } \frac{1}{s}=\frac{1}{p}-\frac{\beta}{n}>0 .
$$


Note. Theorem A has also been proved independently by Jaak Peetre in a paper to appear which, in addition, treats questions on the summability of Fourier integrals [personal communication].

We briefly sketch the proof of A. The method uses complex interpolation as explained, for instance, in [1], [2]. The interpolation space denoted in [2] by $[A, B, \delta(\theta)]$ we will denote by $[A, B]_{\theta}$, or simply by $[A, B]$. Let us further agree to denote the norm of an element $f$ in a Banach space $A$ by $A(f)$.

The proof of Theorem $A$ is facilitated by the change of variable $t=\log |x|, \theta=x /|x|$. The mapping $T: x \rightarrow(\theta, t)$ maps $R^{n}-\{0\}$ onto the cylinder $C=S_{n-1} \times R_{1}$, and functions $f(x)$ are transplanted on $C: f(x)$ $=f^{*}(\theta, t)$. We denote by $C_{t}$ the section of $C:\{\theta, \tau:|\tau-t|<1\}$ and by $H_{m, p, C_{0}}\left(k^{*}\right)$ the Sobolev norm of $k^{*}$ over $C_{0}$ (i.e., the $L^{p}$ norm over $C_{0}$ of $k^{*}$ and all derivatives of orders $\leqq m$. For nonintegral $m>0$ define this norm by interpolation. Set $H_{m, p, c_{t}}\left(k^{*}(\cdot, \cdot)\right) \equiv H_{m, p, c_{0}}\left(k^{*}(\cdot, \cdot+t)\right)$, and $\tilde{H}_{m, p}(k) \equiv \sup _{-\infty<t<\infty} H_{m, p, c_{t}}\left(k^{*}\right)$. Condition (1) is equivalent to

$$
\widetilde{H}_{v, 2}(k)<\infty \quad \text { for some } \nu>\frac{n}{2}
$$

and condition (2) to

$$
\tilde{H}_{r, q}(k)<\infty \text { for some } \nu>\frac{n}{q} .
$$

Thus Theorem A asserts

$$
\text { if (4) holds then } k \in M_{p} \text { for }\left|\frac{1}{2}-\frac{1}{p}\right| \leqq \frac{1}{q} .
$$

We now state a series of lemmas used in the proof.

Lemma 1. If (4) holds for some real $\nu>n / 2$ then $k \in M_{p}$ for all $1<p<\infty$. (This follows by slightly modifying Hörmander's proof.)

LEMMA 2.

$$
\left[H_{m_{1} p_{1}}, H_{m_{2} p_{2}}\right]_{\theta}=H_{m p}
$$

where $m=(1-\theta) m_{1}+\theta m_{2}, 1 / p=(1-\theta) / p_{1}+\theta / p_{2}$ and $1<p_{1}, p_{2}<\infty$.

LEMma 3. Same with $\tilde{H}$ spaces.

Lemma 4. Suppose $A_{1}, B_{1}, A_{2}, B_{2}$ are Banach spaces and $A_{1} \subset B_{1}$, $A_{2} \subset B_{2}$ with corresponding inequalities for the norms, then

$$
\left[A_{1}, A_{2}\right] \subset\left[B_{1}, B_{2}\right]
$$


with the corresponding inequalities for norms.

Lemma 5. $\left[M_{p_{1}}, M_{p_{2}}\right] \subset M_{p}, 1 / p=(1-\theta) / p_{1}+\theta / p_{2}$, with corresponding inequalities for norms.

LEMma 6. It suffices to prove Theorem A for all $p$ in the open interval $|1 / 2-1 / p|<1 / q$.

Proof of Theorem A. We take $\theta=2 / q$, then for sufficiently small $\epsilon>0$, we have, for $|1 / 2-1 / p|<1 / q$,

$$
M_{p} \supset\left[M_{2}, M_{1 / \epsilon}\right] \supset\left[\tilde{H}_{2 \epsilon, n / \epsilon}, \tilde{H}_{n / 2+e, 2}\right]=\tilde{H}_{\alpha, \beta} \supset \tilde{H}_{v, q}
$$

where $\alpha=2 \epsilon(1-2 / q)+2(n / 2+\epsilon) / q, \quad 1 / \beta=\epsilon(1-2 / q) / n+1 / q$ and $\nu>n / q$.

Theorem B follows from Theorem A by considering the operator $K_{1}$ with multiplier $k_{1} \equiv r^{-\beta} k$.

It may seem of little use to know Theorem A for fractional $\alpha$. However we have the following consequence of Theorem $A$ which does not involve fractional derivatives.

THEOREM C. Suppose $k$ is bounded and sufficiently smooth for $x \neq 0$ (for the following quantities to make sense) and for some $0<\theta<1$ the quantity

$$
\begin{aligned}
& {\left[R^{-n} \int_{R<|x|<2 R}\left|R^{m_{1}} D^{m_{1}} k\right| q_{1} d x\right]^{(1-\theta) / q_{1}}} \\
& \cdot\left[R^{-n} \int_{R<|x|<2 R}\left|R^{m_{2}} D^{m_{2} k}\right|^{q_{2}} d x\right]^{\theta / q_{2}}
\end{aligned}
$$

is bounded for $R>0$. Assume further that

$$
(1-\theta)\left(m_{1}-\frac{n}{q_{1}}\right)+\theta\left(m_{2}-\frac{n}{q_{2}}\right)>0 .
$$

Then $k \in M_{p}$ for $|1 / 2-1 / p| \leqq(1-\theta) / q_{1}+\theta / q_{2}$. (In the above, $D^{m} d e-$ notes a generic derivative of order $m$.)

\section{BIBLIOGRAPHY}

1. A. P. Calderon, Intermediate spaces and interpolation, Studia Math. (Ser. Specjalna) Zeszyt 1 (1963), 31-34.

2. J. L. Lions, Construction d'espaces d'interpolation, C.R. Adad. Sci. Paris 251 (1960), 1851-1855.

3. L. Hörmander, Translation invariant operators, Acta Math. 104 (1960), 93-139.

4. S. G. Mikhlin, On the multipliers of Fourier transforms, Dokl. Akad Nauk SSSR (N.S.) 109 (1956), 701-703.

UNIVERSITY OF MINNESOTA 